\title{
A!
}

This is an electronic reprint of the original article.

This reprint may differ from the original in pagination and typographic detail.

Mäkinen, S.; Puska, M. J.

\section{Positron states at vacancy-impurity pairs in semiconductors}

Published in:

Physical Review B

DOI:

10.1103/PhysRevB.40.12523

Published: 15/12/1989

Document Version

Publisher's PDF, also known as Version of record

Please cite the original version:

Mäkinen, S., \& Puska, M. J. (1989). Positron states at vacancy-impurity pairs in semiconductors. Physical

Review B, 4O(18), 12523-12526. https://doi.org/10.1103/PhysRevB.40.12523

This material is protected by copyright and other intellectual property rights, and duplication or sale of all or part of any of the repository collections is not permitted, except that material may be duplicated by you for your research use or educational purposes in electronic or print form. You must obtain permission for any other use. Electronic or print copies may not be offered, whether for sale or otherwise to anyone who is not an authorised user. 


\title{
Positron states at vacancy-impurity pairs in semiconductors
}

\author{
S. Mäkinen \\ Department of Physics, University of Jyväskylä, SF-40100 Jyväskylä, Finland \\ M. J. Puska \\ Laboratory of Physics, Helsinki University of Technology, SF-02150 Espoo, Finland
}

(Received 26 June 1989)

\begin{abstract}
Positron states at pure monovacancies and divacancies and vacancy-phosphorus pairs in $\mathrm{Si}$ as well as at As vacancies and As-vacancy-As-antisite pairs in GaAs are calculated. The dependence of the positron lifetime on the lattice relaxation around the defects is studied, and the effects related to the screening of positrons are discussed. The calculations are based on superimposing free atoms. The ability of the method to describe positron states at charged defects is demonstrated.
\end{abstract}

The positron lifetime technique ${ }^{1,2}$ has proved to be a unique tool in investigating atomic-size defects in metals. Recently the method has been widely applied also in studies of vacancy-type defects and their interactions in semiconductors. ${ }^{3,4}$ However, in the case of semiconductors the positron lifetime spectra are often quite difficult to analyze. This is mainly due to the large number of various point defects: different types of vacancies, interstitials, and antisites at different charge states. Theoretical calculations are thus needed to increase the knowledge of positron-defect interactions in semiconductors.

In this report we present theoretical results for the annihilation characteristics of positrons localized at pure vacancies and at vacancy-phosphorus pairs $\left[V_{\mathrm{Si}}(\mathrm{P})\right]$ in $\mathrm{Si}$. The calculations for positrons at a pure As vacancy $\left(V_{\text {As }}\right)$ and at an As-vacancy-As-antisite pair $\left(V_{\mathrm{As}} \mathrm{As}_{\mathrm{Ga}}\right)$ in $\mathrm{GaAs}$ will also be discussed. The present calculations complement an already published series of theoretical calculations for positron states in semiconductors. ${ }^{5-7}$ Previously it has been shown that the calculations can reproduce the positron lifetime in a perfect lattice within the experimental uncertainty. ${ }^{7}$ It is therefore expected that the comparison of the calculated positron lifetimes with the experimental ones can yield detailed information about the structure (especially the open volume) of the defects. Now the main interest is to study under what terms the theoretical positron lifetime calculations for the different types of defects agree with assignments made on the basis of positron lifetime measurements. In this comparison the theoretical and experimental knowledge about the ionic relaxation around the defect plays an important role.

The calculation of the positron annihilation rates proceeds usually along the following steps. ${ }^{8}$ (i) First the electron density of the host system is calculated without the influence of the positron. (ii) Thereafter the potential for the positron is constructed within the local-density approximation (LDA) as the sum of the Coulomb potential due to nuclei and electrons, and a correlation potential describing the electron-positron correlation effects. (iii) The solution of the corresponding Schrödinger equation gives then the positron energy eigenvalue and the wave function. (iv) Finally, the annihilation rate $\lambda$ or the positron lifetime $\tau$ is calculated from the overlap of the positron and electron densities as

$$
1 / \tau=\lambda=\int d \mathbf{r}\left|\Psi^{+}(\mathbf{r})\right|^{2}\left[\lambda_{v}\left(n_{v}(\mathbf{r})\right)+\lambda_{c}\left(n_{c}(\mathbf{r})\right)\right] .
$$

Here $n_{v}$ and $n_{c}$ are the valence and core electron densities, $\lambda_{v}$ and $\lambda_{c}$ the local annihilation rates with the valence and core electrons, both respectively, and $\Psi^{+}$is the positron wave function.

The formulas for $\lambda_{v}$ and $\lambda_{c}$ take into account the pileup of electrons around the annihilating positron (enhancement). For the core annihilation rate the independent particle model with the constant enhancement of 1.5 is used, whereas in the case of valence electrons the enhancement is a function of the electron density. For a metal host the strong enhancement due to the free conduction-band electrons leads to the Brandt-Reinheimer interpolation formula: ${ }^{9}$

$$
\lambda_{v}^{m}\left(n_{v}(\mathbf{r})\right)=2+134 n_{v}(\mathbf{r})
$$

in $\mathrm{ns}^{-1}$. For semiconductors the enhancement is smaller due to the reduced screening, and is described by the recent model of Puska et al.: ${ }^{7}$

$$
\lambda_{v}^{\mathrm{sc}}\left(n_{v}(\mathbf{r})\right)=2\left[1-\frac{1}{\epsilon_{\infty}}\right]+134 n_{v}(\mathbf{r})
$$

in $\mathrm{ns}^{-1}$, where $\epsilon_{\infty}$ is the high-frequency dielectric constant.

According to the two-component density-functional theory ${ }^{10}$ the above scheme is exact for delocalized positron states (apart from the LDA's) if the electron density is calculated self-consistently at step (i). In the case of a localized state the trapped positron raises the average electron density in its vicinity, and the electron and positron densities should be calculated simultaneously and self-consistently. However, two-component calculations ${ }^{10}$ for model systems have shown that the positron 
annihilation rate is only slightly changed if the above scheme is used instead of the full simultaneous selfconsistency. Moreover, one can still go one step further and use non-self-consistent electron densities obtained by superimposing free atoms ${ }^{11}$ at stage (i). The positron lifetimes calculated with the superimposed electron densities and the ones computed with the self-consistent densities obtained by the linearized muffin-tin orbital (LMTO) band-structure method within the atomic-sphere approximation (ASA) agree within a few picoseconds. ${ }^{5,6}$ This reflects the fact that the positron distribution has a tendency to follow the electron density when the latter is subject to a moderate rearrangement. In the present work we therefore use the practical calculation method based on atomic superposition. The method uses fully three-dimensional charge densities and also the calculated positron wave function is fully three dimensional. The way of constructing the electron density makes it easy to take into account also the ionic relaxation, which indeed has a strong effect on the positron lifetime through the changes in the open volume seen by the positron.

One of the main results of the previous LMTO-ASA calculations ${ }^{5}$ for the vacancies in semiconductors is that the position lifetime has only a weak dependence on the vacancy charge state when the changes in the ionic relaxation are omitted. In order to test if the method of superimposed atoms can reproduce this result, calculations for charged unrelaxed vacancies in $\mathrm{Si}$ were made using the following model: If the charge state of the vacancy is $Z$, the four nearest-neighbor $\mathrm{Si}$ atoms around the vacancy are replaced by $\mathrm{Si}$ ions of charge $(\mathrm{Ze} / \epsilon) / 4$, where $\epsilon$ is the static dielectric constant. The electron density and the potential of the ion were calculated self-consistently using a fractional electron occupation number for the $\mathrm{Si}$ $3 p$ state. Thus, also the potential sensed by the positron changes. The replacement describes the accumulation of electrons into the bound deep levels induced by the vacancy. The calculations were performed for $V_{\mathrm{Si}}^{2-}, V_{\mathrm{Si}}^{-}$, $V_{\mathrm{Si}}^{0}, \quad V_{\mathrm{Si}}^{+}$, and $V_{\mathrm{Si}}^{2+}$ (although the negative- $U$ phenomenon $^{12}$ prevents the existence of $V_{\mathrm{Si}}^{+}$). The re-

TABLE I. The influence of the vacancy charge state on the annihilation characteristics of positrons at pure $V_{\mathrm{Si}} \cdot \lambda_{v}$ and $\lambda_{c}$ are the positron annihilation rates with the valence and core electrons, respectively. $\tau$ is the positron lifetime and $E_{b}$ the positron binding energy to the defect. The present results are compared with the values calculated by using self-consistent electron structures obtained by the LMTO-ASA method (Ref. 5) (the values in parentheses).

\begin{tabular}{ccccc}
\hline \hline Defect & $\begin{array}{c}\lambda_{v} \\
\left(\mathrm{~ns}^{-1}\right)\end{array}$ & $\begin{array}{c}\lambda_{c} \\
\left(\mathrm{~ns}^{-1}\right)\end{array}$ & $\begin{array}{c}\tau \\
(\mathrm{ps})\end{array}$ & $\begin{array}{c}E_{b} \\
(\mathrm{eV})\end{array}$ \\
\hline$V_{\mathrm{Si}}^{2-}$ & 3.80 & 0.052 & 260 & 1.1 \\
& & & $(261)$ & $(1.6)$ \\
$V_{\mathrm{Si}}^{-}$ & 3.83 & 0.054 & 258 & 0.61 \\
$V_{\mathrm{Si}}^{0}$ & 3.87 & 0.054 & 255 & 0.39 \\
& & & $(259)$ & $(1.0)$ \\
$V_{\mathrm{Si}}^{+}$ & & & not & bound \\
$V_{\mathrm{Si}}^{2+}$ & & & not & bound \\
& & & $(254)$ & $(0.5)$ \\
\hline \hline
\end{tabular}

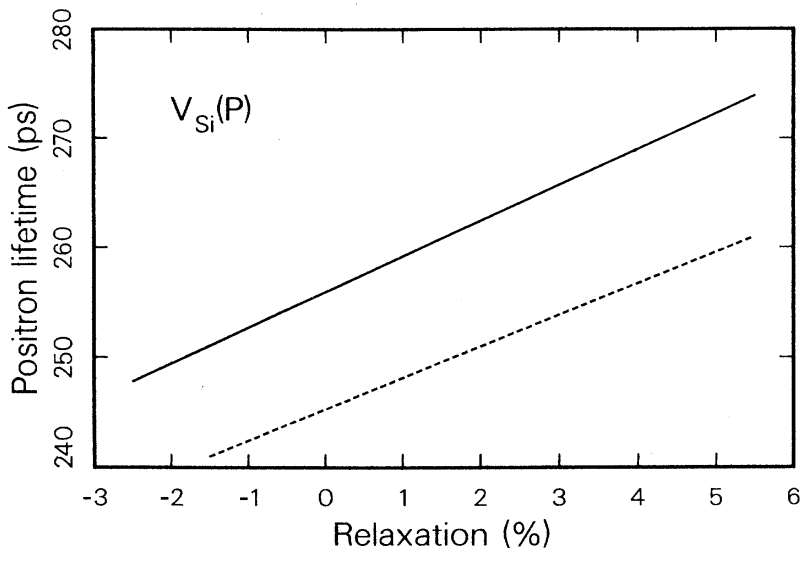

FIG. 1. The positron lifetime in $V_{\mathrm{Si}}(\mathrm{P})$ vs the breathing-mode relaxation around the vacancy. The relaxation is given as the change of the nearest-neighbor bond length relative to the bond length in the perfect lattice. Positive (negative) change indicates outward (inward) relaxation. The solid line corresponds to the results calculated with the semiconductor screening [Eq. (3)] and the dashed line with the metallic screening [Eq. (2)]. The lines for pure $V_{\mathrm{Si}}$ would be $1 \mathrm{ps}$ below these.

sults are qualitatively like the ones obtained by the LMTO-ASA method $^{5}$ (Table I). The positron binding energies are now smaller and the positive vacancies do not bind positrons at all. This supports the observation that positron energetics is sensitive to the self-consistency of the electron density. However, the relative changes in the positron binding energy between different charge states calculated by the two methods are in agreement. This reflects the fact that the relative positions of the energy levels are deterimined by the "extra" charge localized around the vacancy. ${ }^{13}$

According to Table I the present positron lifetimes are in good agreement with the previous ones. Now the changes in the lifetimes are bigger than in the LMTOASA calculations but the increasing trend when the vacancy becomes more negative is well reproduced. This trend results from the increase of negative charge at the vacancy increasing the localization of the trapped positron into the region of lower electron density. This comparison gives credence to our claim that the positron lifetimes can be calculated using non-self-consistent electron densities. The results also show that the change of the vacancy charge state changes the positron lifetime by only a few picoseconds. We thus conclude that the change of the charge state can alter the positron lifetime considerably only through the associated ionic relaxation or through changes in the short-range electronic screening of the positron (enhancement). The change in the screening can also be considered so as to reflect changes in the full simultaneous self-consistency of the electron and positron densities. On the basis of this conclusion we have made the following calculations using only neutral atoms in the superpositions, without trying to describe the slightly different electron charge accumulations in the different charge states of the vacancy. 
The calculated positron lifetime in the perfect Si lattice is $219 \mathrm{ps}$, in excellent agreement with the experimental results. ${ }^{4,14,15}$ The lifetime for positrons localized in a non-

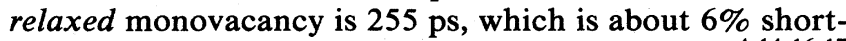
er than the measured lifetimes of $270-275$ ps. $4,14,16,17$ However, acccording to the theoretical electron structure calculations ${ }^{18,19}$ the $\mathrm{Si}$ atoms in the nearest-neighbor positions to the vacancy relax outwards. For example, in the case of the neutral vacancy Baraff et al. ${ }^{18}$ give an outward breathing-mode relaxation of $4.3 \%$ and Scheffler et $a l .{ }^{19}$ of $4.7 \%$. The outward relaxation decreases when the charge state of the vacancy becomes more negative because the "glue" inside the vacancy increases. We can reproduce the experimental positron lifetime of 270 ps by allowing an outward relaxation of $4.2 \%$ (Fig. 1), which is in good agreement with the theoretical estimations given above.

The positron wave function for the case where one of the nonrelaxed nearest-neighbor $\mathrm{Si}$ atoms around the vacancy is replaced by a $\mathrm{P}$ atom ${ }^{20}\left[V_{\mathrm{Si}}(\mathrm{P})\right]$ is shown in Fig. 2. The wave function can hardly be distinguished from that at a pure Si vacancy. ${ }^{6}$ Therefore also the positron lifetime of $256 \mathrm{ps}$ is almost the same as in the pure vacancy. The experimental finding are, however, quite different: a distinctly different lifetime of $248-255$ ps is seen in strongly P-doped Si. ${ }^{14,17}$ This lifetime should correspond to singly negative phosphorus-vacancy pairs. The theoretical explanation of such a short lifetime would ask for an inward relaxation of $0.3-2.5 \%$ (Fig. 1). However, according to the theoretical calculations ${ }^{18,19}$ for the vacancies the direction of the lattice relaxation is believed to be only outwards. Also the isothermal measurements of the hydrostatic pressure dependence on the annealing kinetics of $V_{\mathrm{Si}}(\mathrm{P})$ (Ref. 21) indicate outward relaxations: $6.1 \%$ for the neutral phosphorusvacancy pair and $3.2 \%$ for the singly negative one.

This discrepancy in the case of the phosphorusvacancy pair might mean that the model used for the electron enhancement at the positron [Eq. (3)] is unsatisfactory in this case. The enhancement cannot be larger

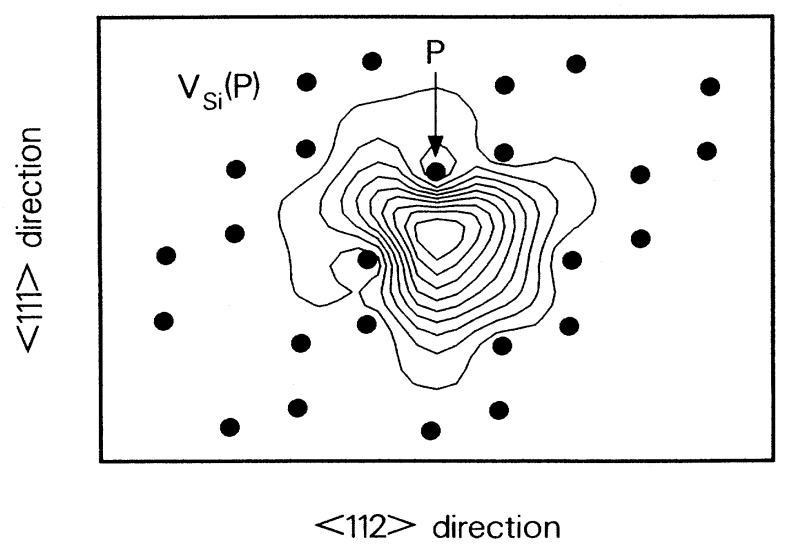

FIG. 2. Positron wave function in $V_{\mathrm{Si}}(\mathrm{P})$. The ion positions are denoted by solid circles. The contour spacing is $\frac{1}{10}$ of the maximum value.

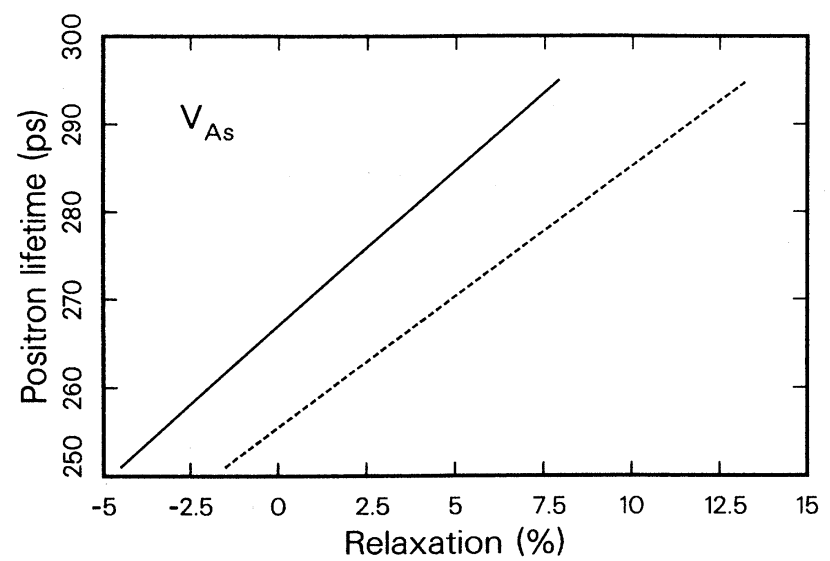

FIG. 3. Same as Fig. 1, but for $V_{\mathrm{As}}$. The lines for $V_{\mathrm{As}} \mathrm{As}_{\mathrm{Ga}}$ would be 1 ps below these.

than that for a metallic system [Eq. (2)]. If the metalliclike screening of positrons is used for $V_{\mathrm{Si}}(\mathrm{P})$, the lifetime would be $246 \mathrm{ps,}$, clearly shorter than that for pure $V_{\mathrm{Si}}$. Now an outward relaxation of $0.9-3.3 \%$ is needed to explain the experimental results (Fig. 1). This lattice relaxation is in agreement with the above-mentioned theoretical and experimental results. The use of the metallic screening for positrons at $V_{\mathrm{Si}}(\mathrm{P})$ can also be justified by the fact that highly P-doped Si has been noticed to have metallic properties. ${ }^{22}$ Also the studies of the Si- $E$ center indicate significant differences in the electronic structure of $V_{\mathrm{Si}}$ and $V_{\mathrm{Si}}(\mathrm{P}) .^{20,23}$

A similar situation arises when there is an As vacancy $\left(V_{\text {As }}\right)$ in GaAs. Also in this case two clearly different lifetimes of about 260 and 295 ps are seen in experiments. ${ }^{24,25}$ The positron lifetime corresponding to this single defect is seen to change between these two values when the Fermi level position crosses a certain ionization level in the band gap. Therefore the longer lifetime is connected to a singly negative vacancy and the shorter to a doubly negative one. ${ }^{24}$ The use of the semiconductor screening results in a positron lifetime of about 267 ps for a nonrelaxed $V_{\text {As }}$, and an inward breathing-mode relaxation of $2.3 \%$ is required to get the 260 ps result (Fig. 3). The situation gets much more reasonable if the metallic screening is used: a $1.3 \%$ outward relaxation brings about the experimentally seen lifetime of 260 ps. Thus the shorter of the experimentally seen defect lifetimes seems to come from positrons annihilating in $V_{\text {As }}$, when the screening of positrons is similar to the screening in metals. In the case of the longer lifetime of 295 ps the use of the semiconductor screening seems to be more appropriate. Metallic screening would ask for an outward relaxation of about $13 \%$, which could be diminished to a more reasonable value of $7.9 \%$ when the semiconductor screening is used. The change in the relaxation of over $10 \%$ between two adjacent charge states is difficult to understand on the basis of the theory for $V_{\mathrm{Si}}$ and the experiments for $V_{\mathrm{Si}}(\mathrm{P})$.

As vacancies do not necessarily exist with ideal lattice surroundings, but form complexes with impurities, inter- 
stitials, or antisites. ${ }^{3,4}$ According to the total enery calculations of Baraff and Schlüter ${ }^{26}$ also the existence of As-vacancy-As-antisite defect $\left(V_{\mathrm{As}} \mathrm{As}_{\mathrm{Ga}}\right)$ is possible in GaAs. We have therefore studied the positron annihilation characteristics for $V_{\mathrm{As}} \mathrm{As}_{\mathrm{Ga}}$, too. As in the case of $V_{\mathrm{Si}}(\mathrm{P})$ the substitution of one of the four atoms nearest to the vacancy by a different type of an atom does not change the positron lifetime very much. The behavior of the lifetime as a function of the lattice relaxation is the same as for the pure $V_{\text {As }}$ in Fig. 3, but the lines would be 1 ps lower.

For divacancies in $\mathrm{Si}\left(V_{\mathrm{Si}} V_{\mathrm{Si}}\right)$ we found that the semiconductor screening seems to work again, because this gives the experimental lifetime of 320 ps (Refs. 16, 27, and 28) with an outward relaxation of $2.8 \%$, which is close to the above-mentioned theoretical and experimental values suggested for $V_{\mathrm{Si}}$ and $V_{\mathrm{Si}}(\mathrm{P})$. The metallic screening does not seem to work for $V_{\mathrm{Si}} V_{\mathrm{Si}}$, because with it a nearly $9 \%$ relaxation should be used.

The preceding examples show that in approximately half of the cases the theoretical calculations with the semiconductor model for annihilation [Eq. (3)] give results which are in reasonable agreement with the positron lifetime measurements and the knowledge about the ionic relaxations. This agreement gives credence to our calculation method based on superimposing free atoms, and it shows, as also the calculations for positron states in perfect semiconductor lattices, that self-consistent electron structures are not necessary for the determination of the positron lifetimes. In order to understand within the present theory the rest of the cases the possible changes in the lattice relaxation around the defect seem to be insufficient. As a possible additional effect it is suggested that also the nature of the screening of positrons is changing. When comparing the isolated Si vacancy and the Si-vacancy-phosphorus pair, which are in the same charge state (i.e., singly negative) the main difference in the electronic structure is that in the former there are three bound deep level electrons whereas their number in the latter is four. Also in the case of the As vacancy or the As-vacancy-As-antisite pair the screening seems to change towards the metallic one when the number of bound electrons at the defect increases. However, one should bear in mind that in the present calculations the effects due to the localized positron either on the ionic or on the electronic structure of the defect have not been taken into account with the exception of the short-range enhancement of the electron density at the positron.

The authors are indebted to Professor M. Manninen, Professor R. M. Nieminen, Professor P. Hautojärvi, and Dr. H. Rajainmäki for many helpful discussions.
${ }^{1}$ Positrons in Solids, Vol. 12 of Topics in Current Physics, edited by P. Hautojärvi (Springer, Heidelberg, 1979).

${ }^{2}$ Positron Solid State Physics, Proceedings of the International School of Physics "Enrico Fermi," Course LXXXIII, Varenna, 1981, edited by W. Brandt and A. Dupasquier (NorthHolland, Amsterdam, 1983).

${ }^{3}$ G. Dlubek and R. Krause, Phys. Status Solidi A 102, 443 (1987).

${ }^{4}$ S. Dannefaer, Phys. Status Solidi A 102, 481 (1987).

${ }^{5}$ M. J. Puska, O. Jepsen, O. Gunnarsson, and R. M. Nieminen, Phys. Rev. B 34, 2695 (1986).

${ }^{6}$ M. J. Puska and C. Corbel, Phys. Rev. B 38, 9874 (1988).

${ }^{7}$ M. J. Puska, S. Mäkinen, M. Manninen, and R. M. Nieminen, Phys. Rev. B 39, 7666 (1989).

${ }^{8}$ For a review, see M. J. Puska, Phys. Status Solidi A 102, 11 (1987).

${ }^{9}$ W. Brandt and J. Reinheimer, Phys. Rev. B 2, 3104 (1970); Phys. Lett. 35A, 109 (1971).

${ }^{10}$ E. Boroński and R. M. Nieminen, Phys. Rev. B 34, 3820 (1986); R. M. Nieminen, E. Boroński, and L. Lantto, ibid. 32 , 1377 (1985).

${ }^{11}$ M. J. Puska and R. M. Nieminen, J. Phys. F 13, 333 (1983).

12J. R. Troxell and G. D. Watkins, Phys. Rev. B 22, 921 (1980).

${ }^{13}$ C. O. Rodriquez, S. Brand, and M. Jaros, J. Phys. C 13, L333 (1980).

${ }^{14}$ J. Mäkinen, C. Corbel, P. Hautojärvi, P. Moser, and F. Pierre, Phys. Rev. B 39, 10162 (1989).

${ }^{15}$ S. Dannefaer, P. Mascher, and D. Kerr, Phys. Rev. Lett. 56, 2195 (1986)
${ }^{16}$ S. Dannefaer, G. W. Dean, D. P. Kerr, and B. G. Hogg, Phys. Rev. B 14, 2709 (1976).

${ }^{17}$ R. Würschum, W. Bauer, K. Maier, A. Seeger, and H.-E. Schaefer, J. Phys: Condensed Matt. (to be published).

${ }^{18}$ G. A. Baraff, E. O. Kane, and M. Schlüter, Phys. Rev. B 21, 5662 (1980).

${ }^{19}$ M. Scheffler, J. P. Vigneron, and G. P. Bachelet, Phys. Rev. B 31, 6541 (1980).

${ }^{20}$ G. D. Watkins and J. W. Corbett, Phys. Rev. 134, A1359 (1964).

${ }^{21}$ C. E. Barnes and G. A. Samara, Mater. Res. Soc. Symp. Proc. 46, 471 (1985); see also G. A. Samara, Phys. Rev. B 37, 8523 (1988).

${ }^{22}$ T. F. Rosenbaum, R. F. Milligan, M. A. Paalanen, G. A. Thomas, R. N. Bhatt, and W. Lin, Phys. Rev. B 27, 7509 (1983).

${ }^{23}$ G. D. Watkins, in Deep Centers in Semiconductors, edited by S. T. Pantelides (Gordon and Breach, New York, 1986).

${ }^{24}$ C. Corbel, M. Stucky, P. Hautojärvi, K. Saarinen, and P. Moser, Phys. Rev. B 38, 8192 (1988).

${ }^{25}$ G. Dlubek, O. Brümmer, F. Plazaola, and P. Hautojärvi, J. Phys. C 19 , 331 (1986).

${ }^{26}$ G. A. Baraff and M. Schlüter, Phys. Rev. B 33, 7346 (1986); Phys. Rev. Lett. 55, 1327 (1985).

${ }^{27}$ W. Fuhs, U. Holzhauser, S. Mantl, F. W. Richter, and R. Sturm, Phys. Status Solidi B 89, 69 (1978).

${ }^{28} \mathrm{~S}$. Dannefaer, in Proceedings of the Eighth International Conference on Positron Annihilation, edited by L. DorikensVanpraet, M. Dorikens, and D. Segers (World Scientific, Singapore, 1989), p. 86. 